\title{
单轴应变对石墨烯掺杂硼、氮、铝、硅、磷的影响与调控
}

\author{
吴其胜 王子路 王金兰*
}

(东南大学物理系 南京 211189)

\begin{abstract}
摘要 掺杂石墨烯因对石墨烯的性质有良好的修饰作用而备受关注. 掺杂石墨烯的实验合成一直都是研究热点, 但有 一个普遍的难题, 就是掺杂困难, 掺杂浓度不高. 针对这一难题, 我们提出了通过对石墨烯施加单轴应变来降低掺杂 过程反应形成能, 从而实现石墨烯的有效可控掺杂的可能性. 我们的第一性原理计算结果表明, 在施加应变时, 拉伸 应变有利于嗍掺杂, 而压缩应变使氮掺杂更容易, 对于铝、硅、磷, 不管是拉伸还是压缩均可以使掺杂更容易. 此外, 我 们还进一步揭示了单轴应变对掺杂石墨烯的电子结构及磁性质的影响规律.
\end{abstract}

关键词 石墨烯; 掺杂; 单轴应变; 第一性原理计算; 电子结构; 磁性

\section{Strain Engineered Modulation on Graphene Doped with Boron, Nitrogen, Aluminum, Silicon and Phosphorus}

\author{
Wu, Qisheng Wang, Zilu Wang, Jinlan* \\ (Department of Physics, Southeast University, Nanjing 211189)
}

\begin{abstract}
Doped graphene has been widely focused because of its modifications on properties of graphene. Syntheses of doped graphene have been hotspots for years and experimentalists are still struggling for obtaining controllable high-quality doped graphene. Usually, rigorous experimental conditions are required. In order to make it easier to dope impurities into graphene, we proposed that uniaxial strain can reduce the formation energy of the process of doping graphene. By using first-principles calculations, we first calculated the formation energy of doping graphene with boron, nitrogen, aluminum, silicon and phosphorus under different uniaxial strains (from $-6 \%$ to $6 \%$ ). After that, we studied the effect of uniaxial strain on the electronic structure and magnetism of doped graphene. The DFT calculations were carried out within the framework of plane-wave density functional theory, implemented in the Vienna ab initio simulation package (VASP). The projector-augmented-wave potentials were employed to describe the electron-ion interaction and the Perdew-Burke-Ernzerhof generalized gradient approximation (PBE-GGA) was used for exchange-correlation functional. The kinetic energy cutoff of 400 $\mathrm{eV}$ was adopted for the plane-wave expansion and the Brillouin zone was sampled by the Monkhorst-Pack scheme. Periodic boundary conditions were used in the simulations, and the graphene sheet was modeled by a rectangular supercell with 60 carbon atoms, indicating that the dosage concentration is $1.67 \%$. We found that uniaxial strain can apparently change the formation energy of doping processes: tensile and compressive strain can reduce the formation energy of graphene doped with boron and nitrogen, respectively, while the formation energy of graphene doped with aluminum, silicon and phosphorus can be reduced by both tensile and compressive strain. The band gaps of doped graphene range from $c a$. 0.1 to $0.9 \mathrm{eV}$ and can be changed by the uniaxial strain, which will reach a maximum under $3 \%$ tensile strain for all the dopant species. Boron-, nitrogen-, aluminum- and silicon-doped graphene are nonmagnetic. On the contrary, phosphorus-doped graphene is magnetic and its magnetic moment decreases under both tensile and compressive strain.
\end{abstract}

Keywords graphene; doping; uniaxial strain; first-principles calculations; electronic structure; magnetism

\section{1 引言}

自从 2004 年曼彻斯特大学的两位科学家 Geim 和 Novoselov 首次在实验上得到单层石墨以来 ${ }^{[1]}$, 近十年 石墨烯一直都是基础及应用科学领域的研究热点. 石墨 烯的 $\mathrm{sp}^{2}$ 杂化结构及离域 $\pi$ 电子使其具有优异的机械性 能 $^{[2] 、}$ 超高的载流子迁移率 ${ }^{[3]}$ 以及热导率 ${ }^{[4]}$ 等特性 ${ }^{[5]}$, 这 些优良的性质使得它在电子电路 ${ }^{[6,7]}$ 、太阳能电池 ${ }^{[8]}$ 、医
学 ${ }^{[9]}$ 等领域有着广阔的应用前景.

纯净石墨烯虽然有良好的性能，但是石墨烯带隙为 零, 因而不能直接应用于电子器件的逻辑电路中. 打开 石墨烯带隙的方式有很多种 ${ }^{[10]}$, 包括化学掺杂 ${ }^{[1]}$ 、施加 应变 ${ }^{[12]} 、$ 把二维石墨烯剪裁成一维石墨烯纳米带 ${ }^{[13]}$ 、吸 附杂质 ${ }^{[14]}$ 、制备特定图案的石墨烯 ${ }^{[15]}$ 等. 化学掺杂通过 其他种类的原子替代碳原子, 破坏 $\mathrm{sp}^{2}$ 杂化, 改变体系

* E-mail: jlwang@seu.edu.cn

Received September 24, 2014; published November 17, 2014.

Supporting information for this article is available free of charge via the Internet at http://sioc-journal.cn.

Project supported by the National Basic Research Program of China (Nos. 2010CB923401, 2011CB302004), the National Natural Science Foundation of China (Nos. 21173040, 21373045) and the Natural Science Foundation of Jiangsu Province (No. BK20130016) in China.

项目受 973 计划(Nos. 2010CB923401, 2011CB302004)、国家自然科学基金(Nos. 21173040, 21373045)和江苏省自然科学基金(No. BK20130016)资助. 
结构，从而对石墨烯的性质进行修饰. 除了打开带隙， 掺杂石墨烯还可以用于吸附小分子 ${ }^{[16]}$, 并作为传感器 使用 ${ }^{[17]}$. 此外, 掺杂石墨烯还可以用作场效应晶体 管 ${ }^{[18]}$ 、电催化剂 ${ }^{[19]}$ 以及锂离子电池 ${ }^{[20]}$ 等等.

实验上, 可以通过化学气相沉积等方法 ${ }^{[21 ~ 23]}$ 合成 掺杂石墨烯，也可以在通过机械剥离获得的石墨烯基础 上, 采用电热或者退火等方法得到掺杂石墨烯 ${ }^{[24 ~ 28]}$. 第 二种方法更容易控制掺杂过程, 得到高质量掺杂石墨 烯. 然而, 在石墨烯中掺入杂质原子有一定难度, 对实 验条件要求较高. 为了降低石墨烯掺杂难度, 本文基于 第一性原理的密度泛函理论, 研究了给石墨烯施加应变 对石墨烯掺杂硼、氮、铝、硅、磷等元素的影响. 计算 表明: 施加应变可以有效改变掺杂过程的反应形成能, 拉伸应变可以使硼更容易掺入石墨烯，压缩应变则可以 使氮掺杂更容易, 而拉伸和压缩应变均可以使铝、硅、 磷掺杂更容易. 另外, 施加应变还可以有效改变石墨烯 的带隙, 拉伸应变为 3\%时带隙达到最大值; 而且, 磷掺 杂会在石墨烯中引入磁性, 且磁矩会随着施加拉伸或压 缩应变的增大而减小.

\section{2 结果与讨论}

\section{1 掺杂石墨烯的结构}

选取的掺杂石墨烯模型为石墨烯矩形超胞, 总共包 含 60 个原子, 如图 1(a)所示. 掺杂计算在该超胞中替代 掺入一个杂质原子, 掺杂浓度为 $1.67 \%$. 由于硼原子、 氮原子的原子半径与碳原子半径相近(表 1), 嗍原子和 氮原子掺入石墨烯后, 掺杂石墨烯仍维持平面结构, 如 图 1(b)所示. 而铝、硅、磷原子掺入石墨烯后, 掺杂石 墨烯已不能维持平面结构, 杂质原子突出到石墨烯平面 外, 如图 1(c)所示, 这是因为铝、硅、磷原子半径要比 碳原子半径大很多(表 1), 杂质原子已无法嵌入原平面 结构中. 随着拉伸应变增大, 杂质原子的高度降低, 掺 杂石墨烯向平面结构趋近, 相反地, 压缩应变越大, 杂 质原子突出到平面外越明显, 杂质原子与石墨烯平面的 高度差随单轴应变改变基本呈线性变化, 如图 1(d)所示.

\section{2 应变对石墨烯掺杂反应形成能的影响}

为了研究应变对石墨烯掺杂难易程度的影响, 我们 计算了硼、氮、铝、硅、磷掺杂石墨烯的反应形成能, 如 图 2 所示. 在计算形成能的过程中, 采用下面的计算公 式

$$
\begin{gathered}
\text { Graphene }+\mathrm{B}^{*} \rightarrow \mathrm{BG}+\mathrm{C}^{*} \\
\text { Graphene }+\mathrm{N}^{*} \rightarrow \mathrm{NG}+\mathrm{C}^{*} \\
\text { Graphene }+\mathrm{Al}^{*} \rightarrow \mathrm{AlG}+\mathrm{C}^{*} \\
\text { Graphene }+\mathrm{Si}^{*} \rightarrow \mathrm{SiG}+\mathrm{C}^{*} \\
\text { Graphene }+\mathrm{P}^{*} \rightarrow \mathrm{PG}+\mathrm{C}^{*} \\
E(\text { 反应形成能 })=E(\mathrm{DG})+E\left(\mathrm{C}^{*}\right)-E(\text { Graphene })-E\left(\mathrm{D}^{*}\right)
\end{gathered}
$$
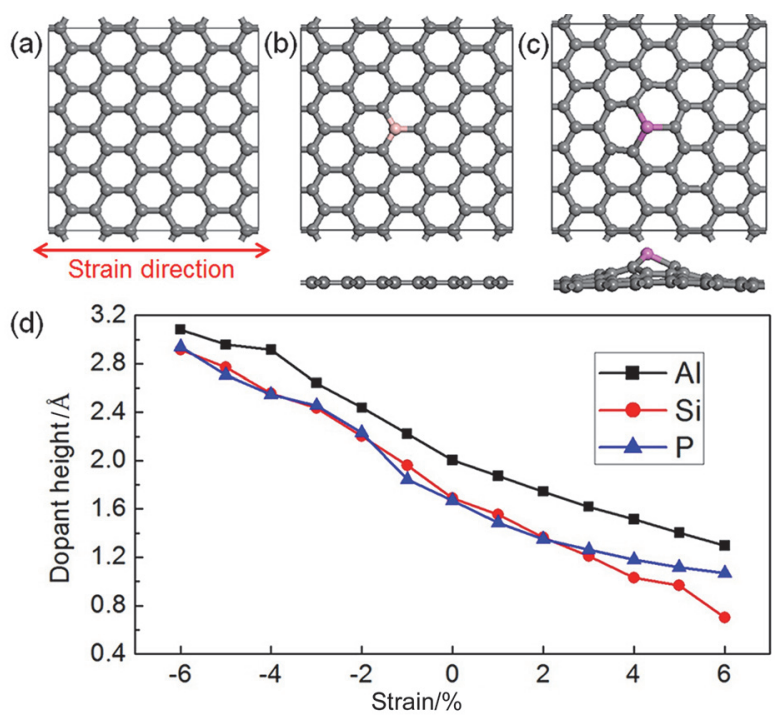

图 1 无应变条件下优化后的石墨烯和掺杂石墨烯结构: (a)石墨烯矩 形超胞(Graphene, 含 60 个碳原子), 红色箭头表示应变方向; (b)嗍、氮 掺杂石墨烯(BG, NG, 俯视图和侧视图); (c)铝、硅、磷掺杂石墨烯(AlG, $\mathrm{SiG}, \mathrm{PG}$, 俯视图和侧视图); (d)铝、硅、磷掺杂石墨烯中杂质原子与石 墨烯平面高度差

Figure 1 Optimized structures of graphene and doped graphene without strain: (a) Graphene rectangle supercell (60 carbon atoms), the red arrow represents the direction of strain; (b) Boron- and nitrogen-doped graphene (BG and NG, top and side view); (c) Aluminum-, silicon- and phosphorus-doped graphene (AlG, SiG and PG, top and side view); (d) The height difference between impurity atom and graphene plane for aluminum-, silicon- and phosphorus-doped graphene

其中 $E(\mathrm{DG})$ 表示掺杂石墨烯的总能, $E$ (Graphene)表示掺 杂前石墨烯的总能, $E\left(\mathrm{C}^{*}\right)$ 和 $E\left(\mathrm{D}^{*}\right)$ 分别表示单个碳原子 及单个杂质原子的能量. 计算得到的形成能为正，表示 掺杂过程需要外界提供能量, 计算得到的形成能为负, 表示掺杂过程对外释放能量. 形成能的大小可以用来衡 量掺杂的难易程度.

从图 2 中可以看出, 各掺杂过程反应形成能均为正, 数值越大, 表明杂质掺入石墨烯的难度越大. 硼、氮掺 杂的反应形成能约为 $2 \sim 4 \mathrm{eV}$, 掺杂相对容易, 而铝、 硅、磷掺杂则较为困难，反应形成能则达到 $6 \sim 10 \mathrm{eV}$.

在给石墨烯施加应变的情况下, 硼、氮掺杂的反应 形成能随应变呈线性变化. 对于硼掺杂石墨烯而言, 随 着拉伸应变的增大，反应形成能减小，而压缩应变会使 反应形成能增大. 氮掺杂石墨烯反应形成能随应变的变 化规律恰好与硼掺杂石墨烯相反. 完全不同于硼、氮掺 杂, 施加拉伸应变或者压缩应变都将会使得铝、硅、磷 掺杂的反应形成能减小, 且应变越大, 反应形成能越小, 即杂质原子更容易掺入石墨烯中. 而且, 压缩应变对 铝、硅、磷掺杂石墨烯反应形成能的影响要比拉伸应变 更明显.

嗍、氮、铝、硅、磷掺杂石墨烯反应形成能随应变 的变化规律与它们的原子半径大小有关. 表 1 给出了碳 原子及各杂质原子的原子半径. 显然, 嗍、氮的原子半 
表 1 硼、碳、氮、铝、硅、磷的原子半径(碳原子半径为 $0.70 \AA$ ). 对应掺杂石墨烯的反应形成能(FE)、带隙(Gap)和磁矩 $(\mu)$.

Table 1 The atom radius of boron, carbon, nitrogen, aluminum, silicon and phosphorus (The radii of carbon atom is $0.70 \AA$ ). Corresponding formation energy (FE), band gap (Gap) and magnetic moment $(\mu)$ of doped graphene

\begin{tabular}{|c|c|c|c|c|c|c|c|c|c|c|c|}
\hline \multirow{3}{*}{$\begin{array}{c}\text { Atom radii } / \AA \\
\text { Strain } / \% \\
\end{array}$} & \multicolumn{2}{|c|}{ B } & \multicolumn{2}{|c|}{$\mathrm{N}$} & \multicolumn{2}{|c|}{$\mathrm{Al}$} & \multicolumn{2}{|c|}{$\mathrm{Si}$} & \multicolumn{3}{|c|}{$\mathrm{P}$} \\
\hline & \multicolumn{2}{|c|}{0.85} & \multicolumn{2}{|c|}{0.65} & \multicolumn{2}{|c|}{1.25} & \multicolumn{2}{|c|}{1.10} & \multicolumn{3}{|c|}{1.00} \\
\hline & $\mathrm{FE}$ & Gap & $\mathrm{FE}$ & Gap & $\mathrm{FE}$ & Gap & FE & Gap & FE & Gap & $\mu$ \\
\hline-6 & 3.38 & 0.21 & 3.38 & 0.06 & 5.06 & 0.05 & 2.31 & 0.09 & 2.32 & 0.63 & 0.76 \\
\hline-5 & 3.28 & & 3.42 & & 6.90 & & 4.12 & & 4.14 & & \\
\hline-4 & 3.17 & & 3.46 & & 8.30 & & 5.49 & & 5.51 & & \\
\hline-3 & 3.06 & 0.05 & 3.5 & 0.11 & 9.27 & 0.17 & 6.43 & 0.18 & 6.45 & 0.73 & 0.82 \\
\hline-2 & 2.95 & & 3.54 & & 9.90 & & 7.02 & & 7.03 & & \\
\hline-1 & 2.85 & & 3.58 & & 10.19 & & 7.27 & & 7.28 & & \\
\hline 0 & 2.73 & 0.26 & 3.61 & 0.28 & 10.27 & 0.26 & 7.30 & 0.29 & 7.31 & 0.83 & 0.83 \\
\hline 1 & 2.64 & & 3.68 & & 10.24 & & 7.23 & & 7.26 & & \\
\hline 2 & 2.55 & & 3.75 & & 10.13 & & 7.07 & & 7.17 & & \\
\hline 3 & 2.44 & 0.41 & 3.80 & 0.39 & 9.94 & 0.40 & 6.86 & 0.40 & 7.04 & 0.92 & 0.61 \\
\hline 4 & 2.34 & & 3.85 & & 9.71 & & 6.60 & & 6.89 & & \\
\hline 5 & 2.23 & & 3.89 & & 9.45 & & 6.33 & & 6.73 & & \\
\hline 6 & 2.13 & 0.15 & 3.94 & 0.17 & 9.16 & 0.25 & 6.04 & 0.09 & 6.56 & 0.81 & 0.50 \\
\hline
\end{tabular}

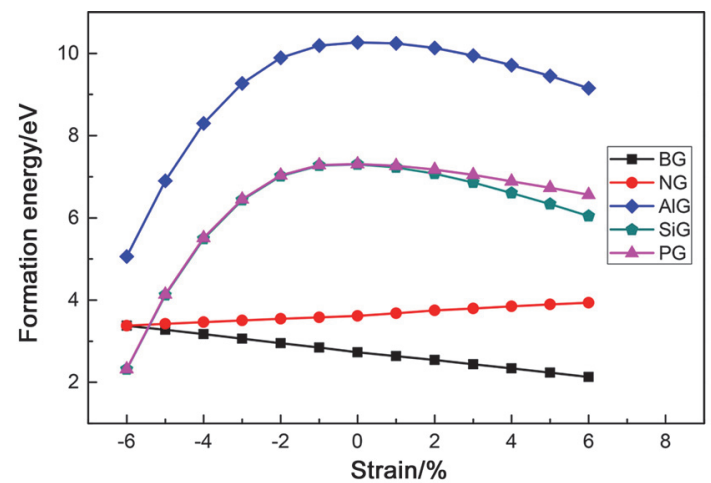

图 2 嗍、氮、铝、硅、磷掺杂石墨烯反应形成能随单轴应变的变化 关系曲线

Figure 2 Formation energy as the function of uniaxial strain for boron-, nitrogen-, aluminum-, silicon- and phosphorus-doped graphene

径与碳原子相近, 掺入石墨烯后填补碳原子的空缺, 整 个体系仍能维持平面结构, 这是硼、氮原子相对容易掺 入石墨烯的原因所在. 由于嗍原子半径大于碳原子半 径, 而氮原子半径小于碳原子半径, 因此拉伸应变使得 嗍原子更容易掺入石墨烯，而压缩应变使得氮原子更容 易掺入石墨烯. 铝、硅、磷原子半径均比碳原子半径大 很多, 相应的掺杂石墨烯已无法维持平面结构, 杂质原 子突出到石墨烯平面外. 施加拉伸应变可以增大空缺间 隙，使得杂质原子更容易掺入石墨烯; 施加压缩应变则 有利于石墨烯凸起. 这两种机制同时作用，导致施加拉 伸应变和压缩应变均可降低铝、硅、磷掺杂的反应形成 能.

我们的进一步计算表明, 沿着石墨烯的 Zigzag 边施 加单轴应变对石墨烯掺杂反应形成能的影响同沿着 Armchair 边施加应变类似, 如 Supporting Information 中
图 S5 所示. 实验上可以首先将石墨烯转移至合适的基 底上, 然后弯曲基底, 由于石墨烯与基底结合紧密, 弯 曲基底相当于给石墨烯施加单轴应变 ${ }^{[29]}$. 鉴于实验上 给石墨烯施加的应变都比较小 ${ }^{[30]}$, 我们在计算中仅考 虑 $-6 \%$ 到 $6 \%$ 的应变. 对于嗍、氮掺杂石墨烯而言, $\pm 6 \%$ 范围内的形变都属于弹性形变, 而对于铝、硅、磷 掺杂石墨烯而言，拉伸应变仍在弹性模量区内，但是压 缩应变属于非弹性形变, 如 Supporting Information 图 S6 所示，因此考虑更大形变没有意义.

\section{3 应变对掺杂石墨烯电子结构的影响}

施加应变可以改变掺杂石墨烯的能带带隙, 如表 1 和图 3 所示. 硼、氮、铝、硅掺杂引起的带隙较小, 均 在 $0.4 \mathrm{eV}$ 以下. 磷掺杂石墨烯引起的带隙较大, 约为

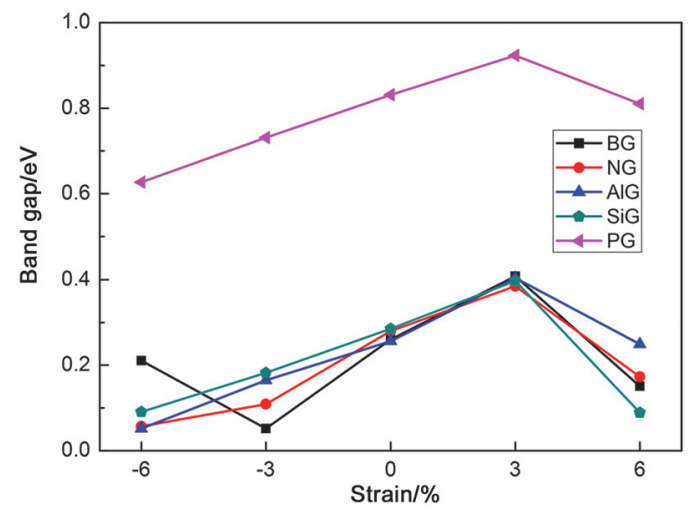

图 3 硼、氮、铝、硅、磷掺杂石墨烯的能带带隙随单轴应变的变化 关系曲线

Figure 3 Band gap as the function of uniaxial strain for Boron-, nitrogen-, aluminum, silicon- and phosphorus-doped graphene 
$0.6 \sim 0.9 \mathrm{eV}$. 从 Supporting information 图 $\mathrm{S} 1 \sim \mathrm{S} 4$ 以及图 4 给出的不同应变下嗍、氮、铝、硅、磷掺杂石墨烯的 分波态密度图可以看出, 硼、氮、铝、硅掺杂石墨烯带 隙相近, 是因为掺杂石墨烯能带带边由石墨烯贡献, 杂 质原子基本无贡献, 且没有引入杂质带. 磷掺杂石墨烯 中, 杂质原子引入杂质带(局域态), 导致能带带隙较大. 从图 3 中可以看出, 应变对带隙有明显的调节作用. 这 五类原子掺杂石墨烯的共同点在于, 施加 $3 \%$ 的拉伸应 变时, 带隙均达到最大值.

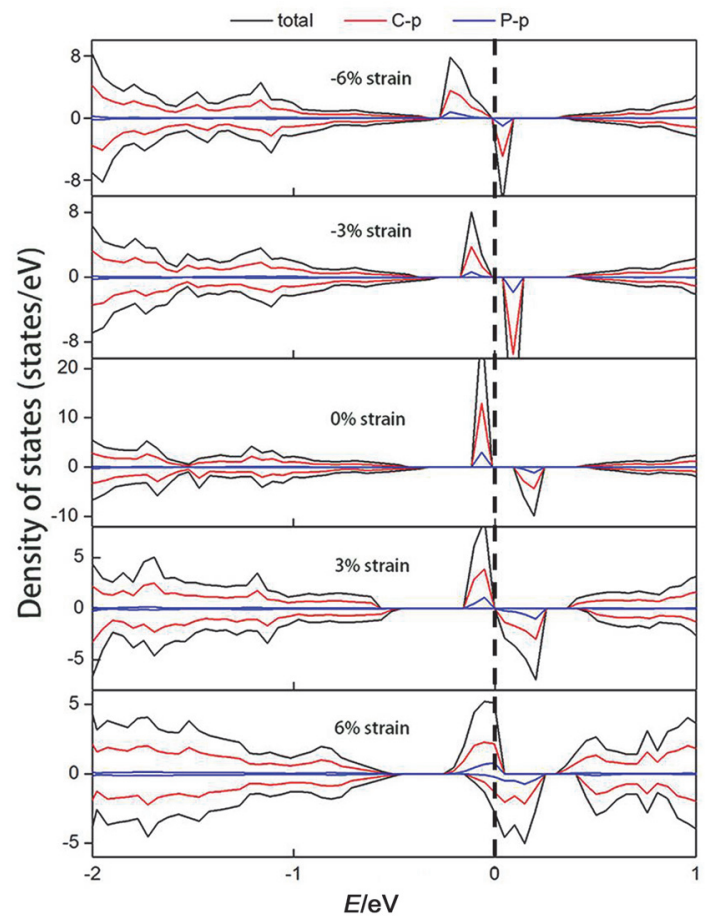

图 4 应变分别为 $-6 \%,-3 \%, 0 \%, 3 \%$ 和 $6 \%$ 时磷掺杂石墨烯的电子 态密度图. total 表示总态密度, C-p 表示碳原子的 $2 \mathrm{p}$ 轨道分波态密度, P-p 表示磷原子的 $3 p$ 轨道分波态密度 (为便于观察, 数值放大为真实 数值 3 倍). 虚线表示费米能级

Figure 4 Density of states of phosphorus-doped graphene for different strains $(-6 \%,-3 \%, 0 \%, 3 \%$ and $6 \%)$. "total" represents total density of states. C-p represents dos projected on $2 p$ orbital of carbon atoms. P-p represents dos projected on $3 p$ orbital of phosphorus atom (data has been multiplied by 3 for clear observation). The dashed line represents the Fermi level

\section{4 应变对掺杂石墨烯磁性的影响}

计算结果表明, 硼、氮、铝、硅掺杂石墨烯均无磁 性, 而磷掺杂石墨烯则具有磁性, 磁矩约为 $0.83 \mu_{\mathrm{B}}$, 与 已有文献结果一致 ${ }^{[31,32]}$. 从态密度图中可以看出, 硼、 氮、铝、硅掺杂石墨烯自旋向上电子与自旋向下电子对 应的态密度完全相同, 且杂质不引入任何局域态. 磷掺 杂石墨烯中, $\pi$ 电子系统的对称性被破坏, 因此对外呈 现磁矩, 且磷掺杂石墨烯的自旋态分布于整个石墨烯超 胞中, 如图 5 所示.

施加拉伸应变或者压缩应变都会减小磷掺杂石墨 烯的磁矩, 如表 1 和图 6 所示. 从磷掺杂石墨烯的自旋
密度分布图(图 5)可以看出，拉伸应变使得自旋密度分 布变得稀疏得多，因此体系磁矩明显减小. 拉伸应变为 $6 \%$ 时磁矩由 $0.83 \mu_{\mathrm{B}}$ 减小至 $0.50 \mu_{\mathrm{B}}$. 由于压缩应变明显 地改变了磷掺杂石墨烯的结构, 因此自旋密度的空间分 布随之发生改变，但是自旋密度大小并没有受太大影 响，因此压缩应变下磷掺杂石墨烯的磁矩无明显改变， 仅略有减小.

$$
\begin{array}{l|l|l} 
& \text { (a) } & \text { (b) } \\
\hline \text { (c) } & \text { (d) } & \text { (e) }
\end{array}
$$
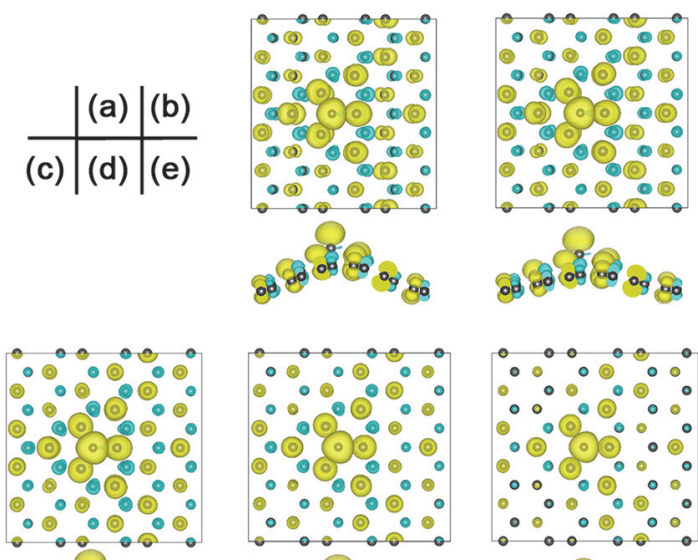

- 80 .
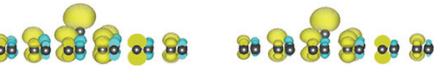

图 5 应变分别为 $-6 \%(a),-3 \%(b), 0 \%(c), 3 \%(d)$ 和 $6 \%$ (e)时磷掺 杂石墨烯的自旋密度分布图. 图中黄色表示电子自旋向上区域, 蓝绿 色表示电子自旋向下区域

Figure 5 Spin density distribution of phosphorus-doped graphene for different strains: $-6 \%$ (a), $-3 \%$ (b), $0 \%$ (c), $3 \%$ (d) and $6 \%$ (e). The yellow areas stand for spin up and the cyan areas stand for spin down

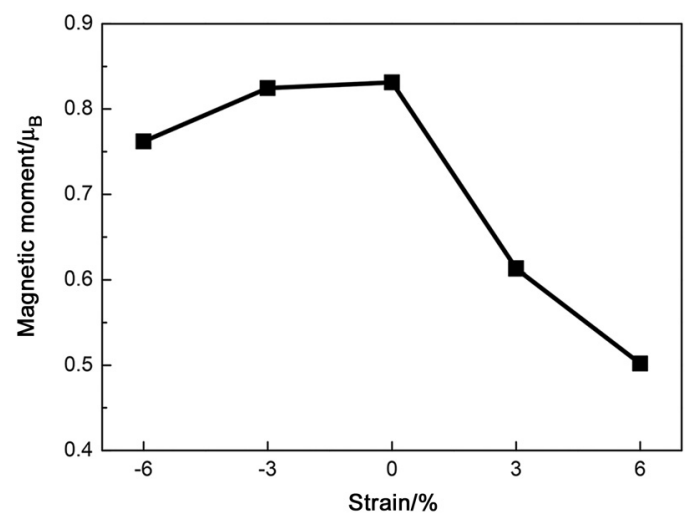

图 6 磷掺杂石墨烯磁矩随单轴应变的变化关系曲线

Figure 6 Magnetic moment of phosphorus-doped graphene as the function of uniaxial strain

\section{3 结论}

基于密度泛函的第一性原理计算, 我们系统研究了 在不同应变条件下，对硼、氮、铝、硅、磷掺杂石墨烯 的形成过程与电磁性质进行了系统研究. 计算结果表 明, 通过对石墨烯施加单轴应变可以有效改变掺杂过程 的反应形成能，拉伸应变可以降低硼掺杂的反应形成 能，压缩应变可以降低氮掺杂的反应形成能，而铝、硅、 
磷掺杂过程反应形成能的降低既可以通过拉伸应变，又 可以通过压缩应变实现. 施加应变还可以引起掺杂石墨 烯能带带隙的变化, 不同应变下各掺杂石墨烯带隙最大 约 $0.9 \mathrm{eV}$, 最小不到 $0.1 \mathrm{eV}$, 施加 $3 \%$ 的拉伸应变可以得 到最大的能带带隙. 嗍、氮、铝、硅掺杂石墨烯无磁性, 而磷掺杂石墨烯具有磁性, 拉伸应变和压缩应变均可使 得磷掺杂石墨烯的磁矩减小, 且拉伸应变对磁矩影响更 明显. 本文理论研究结果表明, 施加适当的应变可以使 石墨烯掺杂过程变得更容易, 且可以对石墨烯的电子结 构及磁性性质进行有效调节, 这为实验上合成可控高质 量掺杂石墨烯提供了有利指导.

\section{4 计算方法与计算模型}

密度泛函理论计算, 是在 VASP (Vienna ab initio Simulation Package $)^{[33]}$ 软件包中进行的. 我们采用投影 缀加波赝势对电子-离子相互作用进行描述, 采用广义 梯度近似(general gradient approximation, GGA) ${ }^{[34]}$ 下的 PBE (Perdew-Burke-Ernzerhof)泛函作为交换关联泛函, 平面波截断能设定为 $400 \mathrm{eV}$. 布里渊区采用 Monkhorst-Pack 特殊 $\mathrm{K}$ 点取样方法 ${ }^{[35]}$ ，总能计算中， $\mathrm{K}$ 点网格取为 $3 \times 3 \times 1$, 态密度计算中, $\mathrm{K}$ 点网格取为 $5 \times$ $5 \times 1$. 选取的计算模型为 $6 \times 5$ 石墨烯矩形超胞, 总共包 含 60 个原子(如图 1 所示). 掺杂计算在该超胞中替代 C 掺入一个杂质原子, 掺杂浓度为 $1.67 \%$, 在实验掺杂浓 度范围之内 (石墨烯掺杂浓度一般为 $1 \% \sim 10 \%{ }^{[36]}$ ). 给掺 杂石墨烯施加的应变为单轴应变, 沿着 armchair 方向, 如图 1(a)所示. 在所有计算中, 真空层大于 $12 \AA$, 从而 保证层间石墨烯的相互作用可以忽略不计. 所有的结构 都是优化到当每个原子受到的最大残余力小于 0.01 $\mathrm{eV} / \AA$ 时截止.

\section{References}

[1] Novoselov, K. S.; Geim, A. K.; Morozov, S. V.; Jiang, D.; Zhang, Y.; Dubonos, S. V.; Grigorieva, I. V.; Firsov, A. A. Science 2004, 306, 666.

[2] Lee, C.; Wei, X.; Kysar, J. W.; Hone, J. Science 2008, 321, 385.

[3] Geim, A. K.; Novoselov, K. S. Nat. Mater. 2007, 6, 183.

[4] Seol, J. H.; Jo, I.; Moore, A. L.; Lindsay, L.; Aitken, Z. H.; Pettes, M. T.; Li, X.; Yao, Z.; Huang, R.; Broido, D.; Mingo, N.; Ruoff, R. S.; Shi, L. Science 2010, 328, 213.

[5] Castro Neto, A. H.; Peres, N. M. R.; Novoselov, K. S.; Geim, A. K. Rev. Mod. Phys. 2009, 81, 109.
[6] Britnell, L.; Gorbachev, R. V.; Geim, A. K.; Ponomarenko, L. A.; Mishchenko, A.; Greenaway, M. T.; Fromhold, T. M.; Novoselov, K. S.; Eaves, L. Nat. Commun. 2013, 4, 1794.

[7] Schwierz, F. Nat. Nanotechnol. 2010, 5, 487.

[8] Wang, J. T.; Ball, J. M.; Barea, E. M.; Abate, A.; Alexander-Webber, J. A.; Huang, J.; Saliba, M.; Mora-Sero, I.; Bisquert, J.; Snaith, H. J.; Nicholas, R. J. Nano Lett. 2014, 14, 724.

[9] Tkacz, R.; Oldenbourg, R.; Mehta, S. B.; Miansari, M.; Verma, A.; Majumder, M. Chem. Commun. 2014, 50, 6668.

[10] Zhang, Y.; Liang, Y. M.; Zhou, J. X. Acta Chim. Sinica 2014, 72, 367. (张芸秋, 梁勇明, 周建新, 化学学报, 2014, 72, 367).

[11] Liu, H. T.; Liu, Y. Q.; Zhu, D. B. J. Mater. Chem. 2011, 21, 3335.

[12] Ni, Z. H.; Yu, T.; Lu, Y. H.; Wang, Y. Y.; Feng, Y. P.; Shen, Z. X. ACS Nano. 2008, 2, 2301.

[13] Han, M.Y.; Özyilmaz, B.; Zhang, Y.; Kim, P. Phys. Rev. Lett. 2007, 98, 206805.

[14] Balog, R.; Jørgensen, B.; Nilsson, L.; Andersen, M.; Rienks, E.; Bianchi, M.; Fanetti, M.; Lægsgaard, E.; Baraldi, A.; Lizzit, S.; Sljivancanin, Z.; Besenbacher, F.; Hammer, B.; Pedersen, T. G.; Hofmann, P.; Hornekær, L. Nat. Mater. 2010, 9, 315.

[15] Dvorak, M.; Oswald, W.; Wu, Z. Sci. Rep. 2013, 3, 2289.

[16] Dai, J.; Yuan, J.; Giannozzi, P. Appl. Phys. Lett. 2009, 95, 232105.

[17] Russell, J.; Král, P. Nano Res. 2010, 3, 472.

[18] Xu, W.; Lim, T.; Seo, H.; Min, S.; Cho, H.; Park, M.; Kim, Y.; Lee, T. Small 2014, 10, 1999.

[19] Li, R.; Wei, Z.; Gou, X.; Xu, W. RSC Adv. 2013, 3, 9978.

[20] Zhang, C.; Mahmood, N.; Yin, H.; Liu, F.; Hou, Y. Adv. Mater. 2013, 25, 4932 .

[21] Xue, Y.; Wu, B.; Jiang, L.; Guo, Y.; Huang, L.; Chen, J.; Tan, J.; Geng, D.; Luo, B.; Hu, W.; Yu, G.; Liu, Y. J. Am. Chem. Soc. 2012, 134,11060

[22] Wu, T.; Shen, H.; Sun, L.; Cheng, B.; Liu, B.; Shen, J. New J. Chem. 2012, 36, 1385.

[23] Cho, Y. J.; Kim, H. S.; Baik, S. Y.; Myung, Y.; Jung, C. S.; Kim, C. H.; Park, J.; Kang, H. S. J. Phys. Chem. C 2011, 115, 3737.

[24] Lin, Y.; Ksari, Y.; Prakash, J.; Giovanelli, L.; Valmalette, J.; Themlin, J. Carbon 2014, 73, 216.

[25] Wang, C. D.; Yuen, M. F.; Ng, T. W.; Jha, S. K.; Lu, Z. Z.; Kwok, S. Y.; Wong, T. L.; Yang, X.; Lee, C. S.; Lee, S. T.; Zhang, W. J. Appl. Phys. Lett. 2012, 100, 253107.

[26] Lin, Y.; Lin, C.; Chiu, P. Appl. Phys. Lett. 2010, 96, 133110.

[27] Guo, B.; Liu, Q.; Chen, E.; Zhu, H.; Fang, L.; Gong, J. R. Nano Lett. 2010, 10, 4975 .

[28] Wang, X.; Li, X.; Zhang, L.; Yoon, Y.; Weber, P. K.; Wang, H.; Guo, J.; Dai, H. Science 2009, 324, 768.

[29] Mohiuddin, T. M. G.; Lombardo, A.; Nair, R. R.; Bonetti, A.; Savini, G.; Jalil, R.; Bonini, N.; Basko, D. M.; Galiotis, C.; Marzari, N.; Novoselov, K. S.; Geim, A. K.; Ferrari, A. C. Phys. Rev. B 2009, 79, 205433

[30] Bissett, M. A.; Tsuji, M.; Ago, H. Phys. Chem. Chem. Phys. 2014, $16,11124$.

[31] Wang, H.; Wang, H.; Chen, Y.; Liu, Y.; Zhao, J.; Cai, Q.; Wang, X. Appl. Surf. Sci. 2013, 273, 302.

[32] Denis, P. A. Chem. Phys. Lett. 2010, 492, 251.

[33] Kresse, G.; Hafner, J. Phys. Rev. B 1993, 48, 13115.

[34] Perdew, J. P.; Burke, K.; Ernzerhof, M. Phys. Rev. Lett. 1996, 77, 3865.

[35] Monkhorst, H. J.; Pack, J. D. Phys. Rev. B 1976, 13, 5188.

[36] Wang, H.; Maiyalagan, T.; Wang, X. ACS Catal. 2012, 2, 781. 